\title{
Differences in phenotype and gene expression of prostate stromal cells from patients of varying ages and their influence on tumour formation by prostate epithelial cells
}

\author{
Yong-Chuan Wang ${ }^{1, *}$, Sheng-Qiang Yu ${ }^{2, *}$, Xiao-Hai Wang ${ }^{1}$, Bang-Min Han ${ }^{1}$, Fu-Jun Zhao ${ }^{1}$, Guang-Hui Zhu ${ }^{3}$, \\ Yan Hong ${ }^{1}$ and Shu-Jie Xia ${ }^{1}$
}

Prostate cancer $(\mathrm{PCa})$ is an age-related disease, and the stromal microenvironment plays an important role in prostatic malignant progression. However, the differences in prostate stromal cells present in young and old tissue are still obscure. We established primary cultured stromal cells from normal prostatic peripheral zone (PZ) of donors of varying ages and found that cultured stromal cells from old donors (PZ-old) were more enlarged and polygonal than those from young donors (PZ-young). Furthermore, based on immunocytochemical and ultrastructural analysis, the components of stromal cells changed from a majority of fibroblasts to a mixture of fibroblasts and myofibroblasts with increasing donor age. Using a three-dimensional in vitro culture system, we found that PZ-old stromal cells could enhance the proliferation, migration and invasion of cocultured benign BPH-1 and PC-3 cells. Using an in vivo tissue recombination system, we also found that PZ-old stromal cells are more effective than PZ-young cells in promoting tumour formation by BPH-1 cells of high passage $(>100)$ and PC-3 cells. To probe the possible mechanism of these effects, we performed cDNA microarray analysis and profiled 509 upregulated genes and 188 downregulated genes in PZ-old cells. Among the changed genes, we found genes coding for a subset of paracrine factors that are capable of influencing adjacent epithelial cells; these include hepatocyte growth factor (HGF), fibroblast growth factor 5 (FGF5), insulin-like growth factor 2 (IGF2), insulin-like growth factor-binding protein 4 (IGFBP4), IGFBP5 and matrix metallopeptidase 1 (MMP1). Changes in the expression of these genes were further confirmed by quantitative real-time polymerase chain reaction (PCR), Western blotting and enzyme-linked immunosorbent assays. Overall, our findings indicate that stromal cells from prostate PZ of old donors are more active than similar cells from young donors in promoting the malignant process of adjacent epithelial cells. This finding hints at a new potential strategy for the prevention of $\mathrm{PCa}$.

Asian Journal of Andrology (2011) 13, 732-741; doi:10.1038/aja.2011.11; published online 6 June 2011

Keywords: coculture; gene expression; peripheral zone; phenotype; prostate cancer; stromal cells

\section{INTRODUCTION}

Prostate cancer (PCa) is the most common malignancy and the second leading cause of cancer-related death in Western men. ${ }^{1}$ Although the precise aetiology of $\mathrm{PCa}$ is still obscure, the prostatic hormonal milieu and age are considered two of the greatest risk factors for $\mathrm{PCa}^{2-5}$ Despite this, the age-associated decline in circulating testosterone and intraprostatic dihydrotestosterone levels seems paradoxical with respect to the higher occurrence of PCa in ageing men. ${ }^{6}$ Furthermore, one extensive analysis of available epidemiological data showed no conclusive evidence for a link between sex steroids and PCa. ${ }^{7}$ Recent increasing evidence has shown that the stromal microenvironment plays an important role in prostatic malignant pathogenesis. ${ }^{8,9}$ Because age-related changes in stromal cells and the possible contribution of such changes to the development of prostatic disease remain poorly understood, further characterisation of the physiological and molecular alterations that occur in prostatic stromal cells of aged individuals is necessary to understand the specific role of the stromal microenvironment in disease.

Primary culture of prostatic cells from surgical specimens offers a valuable model for studying growth regulation and other changes related to prostate disease. In a previous study, we found that cultured stromal cells from the peripheral zone (PZ) and the transitional zone of normal prostate differed in their stroma-epithelium interactions and in their capacity for tumour formation. ${ }^{10}$ However, a comprehensive view of the differences in stromal cells from $\mathrm{PZ}$ of varying ages and of their relative ability to modulate stroma-epithelium interactions is currently lacking.

In this study, we profiled stromal cell cultures from normal prostate PZ, seeking to characterize the age-related phenotype of these cells and to identify specific molecular alterations that might influence

${ }^{1}$ Department of Urology, The Affiliated First People's Hospital of Shanghai Jiao Tong University, School of Medicine, Shanghai 200080, China; ${ }^{2}$ Department of Urology, Yantai Yuhuangding Hospital Affiliated with the Medical College of Qingdao University, Shandong 264000, China and ${ }^{3}$ Department of General Surgery, The Affiliated First People's Hospital of Shanghai Jiao Tong University, Shanghai 200080, China

* These two authors contributed equally to this work.

Correspondence: Professor SJ Xia (xsjurologist@163.com)

Received: 30 September 2010; Revised: 1 December 2010; Accepted: 21 January 2011; Published online: 6 June 2011 
epithelial malignant progression in vitro and in vivo. Our results provide new insights into the biology of the development and progression of prostate malignancies and suggest potential strategies for preventing the development of PCa. ${ }^{8}$

\section{MATERIALS AND METHODS}

\section{Primary culture of prostatic stromal cells and cell lines}

Fresh prostate tissue was obtained, as previously reported, ${ }^{10}$ from normal PZ of five young donors $(23,25,26,30$ and 32 years old) and five old donors $(56,61,64,71$ and 75 years old). All of the donors exhibited serum levels of total prostate-specific antigen in the normal range ( $\leqslant 4 \mathrm{ng} \mathrm{ml}^{-1}$ ). To further assure the 'normal' status of prostate specimens, the adjacent tissue around each specimen was examined by two pathologists after haematoxylin and eosin (H\&E) staining using the procedure reported by Barclay et al. ${ }^{8}$ The tissue specimens were minced into pieces less than $1 \mathrm{~mm}^{3}$ in size and incubated with type I collagenase $\left(200 \mathrm{U} \mathrm{ml}^{-1}\right)$ for $8-10 \mathrm{~h}$, washed on a $149-\mu \mathrm{m}$ filter and then plated in $25-\mathrm{cm}^{2}$ flasks in $5 \mathrm{ml}$ of RPMI 1640 medium supplemented with $10 \%$ fetal bovine serum (FBS) and antibiotics $(100 \mu \mathrm{g}$ $\mathrm{ml}^{-1}$ streptomycin and $100 \mathrm{IU} \mathrm{ml}^{-1}$ penicillin), insulin $\left(10 \mu \mathrm{g} \mathrm{ml}^{-1}\right)$, transferrin $\left(5.5 \mu \mathrm{g} \mathrm{ml}^{-1}\right)$ and sodium selenite $\left(0.67 \mathrm{ng} \mathrm{ml}^{-1}\right)$ at $37^{\circ} \mathrm{C}$ in $5 \% \mathrm{CO}_{2}$. All supplements were purchased from Gibco Invitrogen (Grand Island, NY, USA). Passage- 3 to -5 cells were used in all subsequent experiments. Cell morphology was assessed using an inverted light microscope. All experimental protocols were approved by the Medical Ethics Committee of the Shanghai First People's Hospital (Shanghai, China). BPH-1 and PC-3 cells were kind gifts from Professor Ju Zhang (Institute of Molecular Biology, College of Life Sciences, Nankai University, Tianjin, China).

\section{Ultrastructural analysis by transmission electron microscopy}

Prostatic stromal cells were fixed by immersion in $2 \%$ glutaraldehyde $\left(4{ }^{\circ} \mathrm{C}\right)$, postfixed with $1 \%$ osmium tetroxide for $2 \mathrm{~h}$, dehydrated in a graded acetone series and subsequently embedded in epoxy resin. Ultrathin sections were cut using a diamond knife and stained with $2 \%$ uranium acetate for $30 \mathrm{~min}$, followed by staining with $2 \%$ lead citrate for $10 \mathrm{~min}$. The samples were evaluated under a CM-120 electron microscope at $80 \mathrm{kV}$ (Philip, Eindhoven, The Netherlands).

\section{Immunocytochemistry}

Cells cultured on 24-well chamber coverslips were fixed in 4\% paraformaldehyde for $20 \mathrm{~min}$ and permeabilized in $0.5 \%$ Triton X-100 (Sigma, St Louis, MO, USA) for $10 \mathrm{~min}$ and then incubated with $3 \% \mathrm{H}_{2} \mathrm{O}_{2}$ for $20 \mathrm{~min}$ at $37{ }^{\circ} \mathrm{C}$ and blocked in $5 \%$ bovine serum albumin for $30 \mathrm{~min}$ at room temperature. Monoclonal primary antibodies against prolyl-4-hydroxylase $(1: 80)$ and alpha-smooth muscle actin ( $\alpha$-SMA, $1: 100)$ were added, and the slides were incubated at $4{ }^{\circ} \mathrm{C}$ overnight. After being washed, slides were incubated with FITClinked, TRITIC-linked secondary antibodies. The antibodies were purchased from Long Island Biotech (Shanghai, China). The slides were mounted with nucleus staining solution 4'-6-diamidino-2phenylindole. Microscopic images from five random fields per well were analysed.

\section{Senescence-associated $\beta$-galactosidase assay}

Stromal cells were plated in six-well culture plates $\left(5 \times 10^{4} /\right.$ well $)$. Twenty-four hours later, the cells were washed with phosphatebuffered saline and fixed with $4 \%$ formaldehyde for $15 \mathrm{~min}$ and then washed thrice with phosphate-buffered saline and stained with a solution (senescence-associated $\beta$-galactosidase kit; Beyotime Biotech, Shanghai, China) containing $1.0 \mathrm{mg} \mathrm{ml}^{-1} \mathrm{X}$-gal, $40 \mathrm{mmol} \mathrm{l}^{-1}$ citric acid/ $\mathrm{Na}_{2} \mathrm{HPO}_{4}\left(\mathrm{pH}\right.$ 6.0), $5 \mathrm{mmol}{ }^{-1}$ potassium ferrocyanide, $150 \mathrm{mmoll}^{-1} \mathrm{NaCl}$ and $2 \mathrm{mmoll}^{-1} \mathrm{MgCl}_{2}{ }^{11}$ After incubation at $37^{\circ} \mathrm{C}$ overnight, positive cells were counted under light microscopy at $\times 100$ magnification.

\section{Stromal cell proliferation assay}

Stromal cells from PZ-young and PZ-old donors were seeded at $1 \times 10^{4}$ cells/well in phenol red-free RPMI 1640 with 5\% FBS (0.5 ml/well). The growth rates of the cells were determined using the Cell Counting Kit-8 (CCK-8 assay; Beyotime Biotech). CCK- 8 working solution was added to the cultures on days 1,2, 3, 4 and 5 and the cells were incubated for $2 \mathrm{~h}$ at $37^{\circ} \mathrm{C}$. Absorbance was measured at $490 \mathrm{~nm}$ using a microplate spectrophotometer.

\section{cDNA microarray analysis}

Total RNA was isolated from stromal cells of PZ-young (23, 25 and 30 years) and PZ-old (56, 64 and 71 years) donors using TRIzol reagent (Sigma) and purified using the RNAeasy Mini Kit (Qiagen GmbH, Hilden, Germany) according to the manufacturer's protocol. RNA samples from each group were used to generate biotinylated cRNA targets for the Affymetrix Human U133 Plus 2.0 Array, which contains 39000 transcripts. After hybridisation, fluorescent signal intensities for all spots on the arrays were analysed using the Gene Chip Operating System (GCOS; Affymetrix, Santa Clara, CA ,USA). Signal intensity ratios between PZ-young and PZ-old were calculated. Genes with a fold change $\geqslant 2$ or $\leqslant 0.5$ were chosen for further analysis. Hierarchical cluster analysis was performed to define the gene expression patterns. The selected genes were grouped into functional categories based on information from the Gene Ontology database (http:// www.geneontology.org/).

\section{Quantitative real-time polymerase chain reaction (Q-PCR)}

To validate the microarray data, a subset of differentially expressed genes was selected, and their expression levels were confirmed by Q-PCR on an ABI 7900 instrument using SYBR Green PCR Master Mix (Takara Biotech, Dalian, China) according to the manufacturer's recommendations. The mixture contained $1 \mu \mathrm{l}$ of cDNA template from the reverse transcription reaction, $2.4 \mu \mathrm{l} \mathrm{MgCl}_{2}$ $\left(25 \mathrm{~mm} \mathrm{~mol}{ }^{-1}\right), 0.75 \mu \mathrm{l}$ of each oligonucleotide $\left(10 \mathrm{~mm} \mathrm{~mol}^{-1}\right)$ and $2 \mu \mathrm{l}$ SYBR Green Mix in a total volume of $20 \mu \mathrm{l}$. Following 5 min of initial denaturation, the reactions were subjected to 34 cycles of $10 \mathrm{~s}$ denaturation at $95^{\circ} \mathrm{C}, 20 \mathrm{~s}$ annealing at $60^{\circ} \mathrm{C}$ and $30 \mathrm{~s}$ extension at $72{ }^{\circ} \mathrm{C}$. Primers for target genes were designed using Primer Premier 5.0 software (Premier Biosoft International, Palo Alto CA, USA), which follows all the guidelines specified for PCR primer design; the specific efficiencies of individual primers were measured. The primers used were as follows: human fibroblast growth factor 5 (FGF5): $5^{\prime}$ GTAACCAATCCAGTGAATAGA-3' (sense) and 5' -TATGTCCAGCAGTCAGTAT-3' (antisense); hepatocyte growth factor (HGF): 5'-GCTGACAATACTATGAATGAC-3' (sense) and 5'-TCGTGAGGATACTGAGAAT-3' (antisense); insulin-like growth factor 2 (IGF2): 5'-CGGAACATTGGACAGAAG-3' (sense) and 5'-GGCGAGGCAGAATAT AAC-3' (antisense); insulin-like growth factor-binding protein 4 (IGFBP4): 5'-CGTCCTGTGCCCCAGGGTTCCT-3' (sense) and 5'-GAAGCTTCACCCCTGTCTTCCG-3' (antisense); IGFBP5: 5'-TTCGTTGTTGTTG TTGTTG-3' (sense) and 5'-AGAGTTATGGTATGAATGTATGT-3' (antisense); matrix metallopeptidase 1 (MMP1): 5'-GAGTCACTGATACACAGAATA-3' (sense) 
and $5^{\prime}$-ATGAATCCATAAGCCACAA-3' (antisense); superoxide dismutase 2, mitochondrial: 5'-CACGCTTACTACCTTCAG-3' (sense) and 5'-GACAGGACATTATCTTGCT-3' (antisense); glyceraldehyde3-phosphate dehydrogenase: 5'-TGCACCACCrAACTGCTTAGC-3' (sense) and 5'-GGCATGGACTGTGGTCATGAG-3' (antisense). Sequence detection system software (version 1.6.3; Applied Biosystems, Warrington, UK) was used for real-time data collection and analysis. Each target gene was normalized to glyceraldehyde-3-phosphate dehydrogenase mRNA expression using the $2^{-\Delta \Delta \mathrm{C}_{\mathrm{t}}}$ method. ${ }^{12}$

\section{Western blot analysis}

Stromal cell cultures of $80 \%$ confluence were lysed in RIPA buffer (Beyotime Biotech). The resulting protein samples were electrophoresed and transferred to polyvinylidene difluoride membrane (Millipore, Billerica, MA, USA). The membranes were blocked with $5 \%$ bovine serum albumin for $1 \mathrm{~h}$ and probed overnight at $4{ }^{\circ} \mathrm{C}$ with primary antibodies including mouse anti-human HGF (1:1000; Abcam, Cambridge, UK), mouse anti-human IGF2 (1:1000), mouse antihuman IGFBP5 $(1: 1000)$ and mouse anti-human MMP1 (1:1000). The membranes were then incubated with horseradish peroxidaseconjugated secondary antibodies for $1 \mathrm{~h}$ at room temperature. Mouse anti-human $\beta$-actin $(1: 1000)$ was used as an internal control. Immune complexes were visualized using an enhanced chemiluminescence kit (Millipore, Billerica, MA, USA) and exposure to Biomax film (Kodak, Rochester, NY, USA). Images of blots were scanned, and the optical densities of the protein bands were quantified using Image Pro-Plus 6.0 software (Media Cybernetics, Bethesda, MD, USA).

\section{Enzyme-linked immunosorbent assays (ELISA)}

Stromal cells were seeded in $2 \mathrm{ml}$ of RPMI 1640 medium containing $5 \%$ charcoal-stripped FBS (Gibco Invitrogen) in six-well plates at a density of $2 \times 10^{5}$ cells/well. After $48 \mathrm{~h}$, the supernatants were harvested, centrifuged at $\times 1000 \mathrm{~g}$ for $25 \mathrm{~min}$ at $4{ }^{\circ} \mathrm{C}$ and analysed using commercially available ELISA kits (R\&D Systems, Minneapolis, MN, USA) for HGF, IGF2, IGFBP5 and MMP1, following the instructions of the manufacturer.

\section{Three-dimensional cocultures in vitro}

We cultured cells in a two-chamber coculture system (Costar; Corning Inc., Corning, NY, USA) in which stromal lawns were separated from epithelial cells (BPH-1 or PC-3 cells) by microporous filters ( 0.4 or $8 \mu \mathrm{m}$ pore size). This method prevented direct contact but permitted exchange of soluble factors between the cultures.

\section{Proliferation assays}

Stromal cells from PZ-young and PZ-old donors and two prostate epithelial cell lines, BPH-1 (immortalized but non-tumourigenic) and PC-3 cells, were cultured in transwell plates using an insert with a $0.4-\mu$ m pore filter. $\mathrm{BPH}-1$ or PC- 3 cells $\left(5 \times 10^{3} /\right.$ well $)$ were plated in $0.2 \mathrm{ml}$ basal medium (phenol red-free RPMI 1640 with $0.1 \%$ bovine serum albumin) in the inserts; the stromal cells $\left(2 \times 10^{4} /\right.$ well $)$ were seeded in $0.5 \mathrm{ml}$ complete medium (phenol red-free RPMI 1640 with $5 \%$ charcoal treated FBS) in the lower chamber. The growth rates of the cultures in the upper chamber were determined by CCK- 8 assay for 4 days as described in the section on 'Ultrastructural analysis by transmission electron microscopy'.

\section{Cell migration and invasion assays}

For analysis of the influence of stromal cells on epithelial cell motility, BPH-1 or PC-3 cells $\left(6 \times 10^{4} /\right.$ well $)$ were seeded onto microporous $\left(\begin{array}{lll}8.0 \mu \mathrm{m}\end{array}\right)$ permeable inserts and stromal cells $\left(2 \times 10^{5} /\right.$ well $)$ were seeded in the lower chamber as chemoattractants. The cell invasion assay was conducted as described above except that the cell density in the upper chamber was $1.2 \times 10^{5} /$ well and the microporous inserts were coated with $50 \mu \mathrm{l}$ Growth FactorReduced Matrigel (Becton Dickinson Bioscience, Bedford, MA, USA). After incubation for $12 \mathrm{~h}$, the cells in the upper chamber were removed with a cotton swab. The cell culture inserts were fixed with $4 \%$ paraformaldehyde and stained using crystal violet, and the migrated cells in six representative microscopic fields $(\times 200)$ of the bottom of each filter were counted.

\section{Assessment of tumour formation in athymic mice}

Male nude mice, 6 weeks old, were purchased from the Animal Center of the Chinese Academy of Sciences (Shanghai, China) and raised in a specific pathogen-free barrier facility according to our institutional guidelines. BPH-1 (passage $>100)$ or PC-3 cells $\left(5 \times 10^{6}\right)$ were suspended in $0.1 \mathrm{ml}$ of undiluted Matrigel (Becton Dickinson Bioscience) and inoculated subcutaneously, either alone or mixed in a $1: 1$ ratio with stromal cells from PZ-young and PZ-old tissues (all 10 donor specimens were used individually). The xenografts were placed in the right hindquarters of male athymic mice; each mouse received three xenografts, and five mice were included in each group. Tumour size was measured weekly. The animals were killed with $\mathrm{CO}_{2}$ at the end of the experiment. Harvested tumours were fixed with $10 \%$ formalin and processed by routine H\&E staining and for immunohistochemical expression of Ki67 (S-P technique, counterstaining with diaminobenzidine).

\section{Statistical analysis}

The data are presented as mean \pm s.d. All experimental procedures were performed in triplicate SPSS for Windows (Version 14.0; Chicago, IL, USA) was used to conduct statistical analyses. Data were analysed using paired $t$-test and one-way ANOVA. $P<0.05$ was considered statistically significant.

\section{RESULTS}

Primary culture and characterisation of prostate stromal cells from donors of varying ages

We established primary cultured stromal cells from normal prostatic $\mathrm{PZ}$ of donors of varying ages and found that all cultured cells showed a typical polar spindle shape (Figure $\mathbf{1}$ a1 and a2). However, the cells became more enlarged and flattened with increasing age of the donor (Figure 1 a1 and a2). In immunocytochemical assays, all cell cultures were positive for prolyl-4-hydroxylase (an enzyme specific for fibroblasts) (Figure 1 b1-b3), but almost all were negative for Pan-CK (specific for epithelium) (data not shown). This finding indicates that the purity of the stromal cells was sufficiently high that the cultures could be used in further studies. Double-label fluorescent immunocytostaining for prolyl-4-hydroxylase and $\alpha$-SMA (specific for smooth muscle cells) ${ }^{13,14}$ was used to identify myofibroblasts; such staining was markedly increased in the PZ-old cells, suggesting an age-dependent transition of these stromal cells from fibroblasts to myofibroblasts (Figure 1 b1-b3). Positive immunostaining for SA- $\beta$-GAL was rarely observed in PZ-young cell cultures, but was easily seen in PZ-old cells (Figure 1 c1-c3). The ultrastructural analysis also clearly showed that PZ-old cells displayed an increased number of cytoplasmic organelles, including dilated rough endoplasmic reticulum, Golgi complexes and mitochondria, which are features of myofibroblasts with active cellular synthetic activity (Figure $\mathbf{1} \mathbf{d} \mathbf{1}$ and $\mathbf{d} 2$ ). Prominent bundles of 
PZ-young
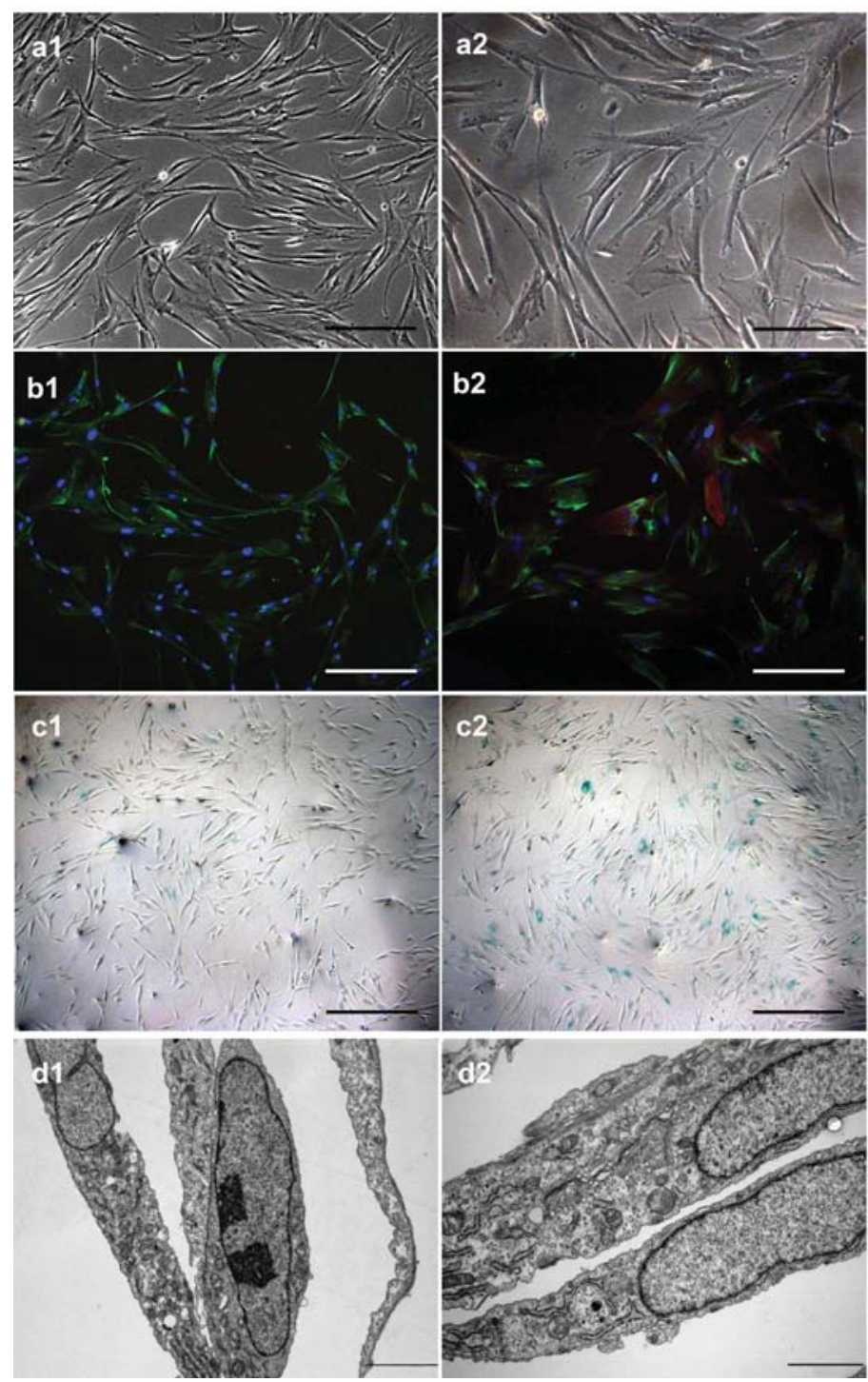

b3

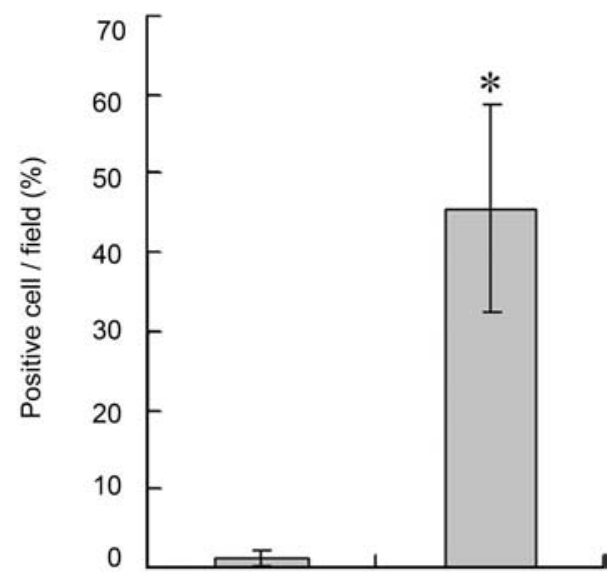

PZ-young

PZ-old

Figure 1 Determination of phenotype and ultrastructure of stromal cell cultures from normal prostate peripheral zone of young and old donors. (a1, a2) The PZ-old stromal cells were generally larger and more polygonal than the PZ-young cells. Scale bars $=50 \mu \mathrm{m}$. (b1-b3) In double-label fluorescent immunocytostaining, all stromal cells were positive for prolyl-4-hydroxylase (green); a significant increase in the percentage (from $1.22 \% \pm 0.97 \%$ to $45.67 \% \pm 13.13 \%, * P<0.01$ ) of $\alpha$-SMApositive cells (red) occurred with increased donor age. Nuclei are stained by DAPI (blue). Scale bars $=50 \mu \mathrm{m}$. (c $1-\mathbf{c} 2) \mathrm{SA}-\beta$-GAL staining showed more positive signals (blue) in PZ-old stromal cells; the quantitative data are shown in $\mathbf{c} 3$. $* P<0.01$. Scale bars $=100 \mu \mathrm{m}$. (d1, d2) TEM ultrastructural analysis of stromal cell cultures derived from PZ-young and PZ-old donors. Compared to PZ-young stromal cells, PZ-old cells display increased dilated rough endoplasmic reticulum, Golgi complexes, prominent bundles of microfilaments and lysosomes in d2. Scale bars $=2 \mu \mathrm{m}$. DAPI, 4'-6-diamidino-2-phenylindole; PZ, peripheral zone; $\alpha$-SMA, alpha-smooth muscle actin.

microfilaments and lysosomes were also regularly observed in cultures of PZ-old but not of PZ-young cells. These results suggest that ageing is an important factor that influences myogenic transdifferentiation of prostate stromal cells.

\section{In vitro growth rate of stromal cell cultures}

Figure 2 shows a comparison of the in vitro growth rates of stromal cell cultures from donors of varying ages. By one-way ANOVA, the stromal cell cultures could be classified into fast- (PZ-23, -26 and -32$)$ and slow-growing groups (PZ-56, -64 and -71). These results indicate that increased age is associated with slower growth of prostate stromal cells in culture.
Stromal cells stimulate the proliferation, migration and invasion of epithelial cells in vitro in an age-related manner

We cocultured BPH-1 and PC-3 prostate epithelial cells with stromal cells in a three- dimensional coculture system and found that both epithelial cell lines grew faster after $48 \mathrm{~h}$ of coculture with PZold cells (Figure 3a and b). To determine whether the stromal cells directly affected the migration and invasion of prostate epithelial cells, we cocultured the cells in a three-dimensional system that allowed assessment of migration and invasion of the cells. Compared with BPH-1 or PC-3 cells alone and with cells from the PZ-young group, stromal cells from the PZ-old samples markedly triggered the migration of $\mathrm{PC}-3$ and $\mathrm{BPH}-1$ cells through the 


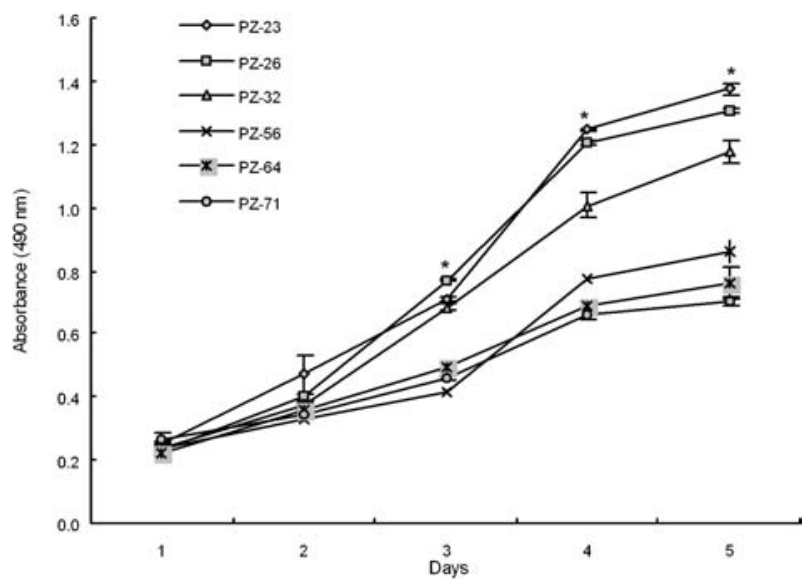

Figure 2 In vitro proliferation rates of human prostatic stromal cell cultures derived from $\mathrm{PZ}$ of varying ages. After day 3 in culture, the average proliferation rate of the young stromal cells (PZ-23, -26 and -32 ) was higher than that of the cells from older donors (PZ-56, -64 and -71$)(* P<0.01)$. PZ, peripheral zone.

uncoated filters (Figure 3c). Using microporous filters coated with GFR-Matrigel to form a barrier between an upper chamber containing epithelial cells and a lower chamber containing stromal cells, we observed that the invasion of PC-3 cells was strongly promoted by stromal cells of the PZ-old group but not by stromal cells of the PZ-young group (Figure 3d). These results suggest that age-related changes in stromal cells can promote the proliferation, migration and invasion of epithelial cells and may thus enhance their malignant progression. a

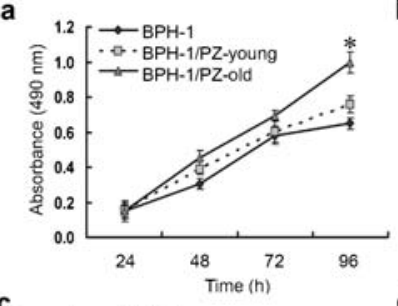

c

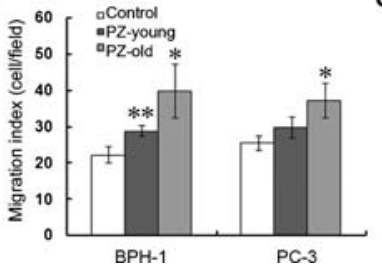

b

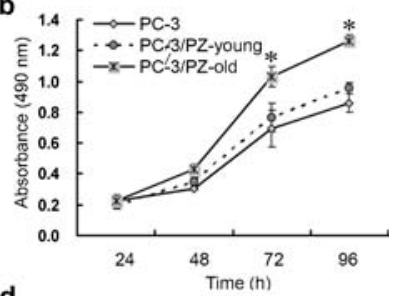

d

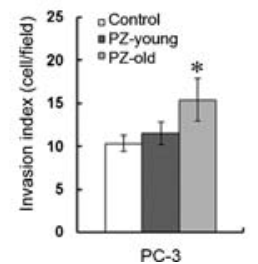

Figure 3 Stromal cells stimulate the proliferation, migration and invasion of prostate epithelial cells in vitro in an age-related manner. Proliferation of $\mathrm{BPH}-1$ (a) and PC-3 (b) in stromal cell-conditioned medium was measured using a CCK-8 assay. Stimulation by stromal cells was performed for $96 \mathrm{~h}$. The mean values of four independent measurements \pm s.d. are shown. $* P<0.01$, compared with other two groups. (c) Migration assays showed that both $\mathrm{BPH}-1$ and $\mathrm{PC} 3$ cells migrated faster when cocultured with PZ-old cells ( $* P<0.01$ : PZ-old groups versus $\mathrm{PZ}$-young or control group; $* * P<0.05$ : PZ-young groups versus control group). (d) Invasion index of PC-3 cells through GFR-MatriGel-coated transwell filters. There was no difference in the invasion index of the PC-3/PZ-young group and that of the PC-3 group; the invasion index of PC-3/PZ-old was significantly higher than those of the other two groups $\left({ }^{*} P<0.01\right)$. CCK-8, Cell Counting Kit-8; PZ, peripheral zone.
Stromal cells enhance tumourigenesis in vivo in an age-related manner

To assess whether the ability of stromal cells to create a microenvironment and promote the tumourigenesis of prostate epithelial cells in vivo is related to the age of the donor from whom the stromal cells are derived, we injected BPH-1 cells into nude mice either alone or with stromal cells from PZ-young or PZ-old donors. We first attempted this experiment using low-passage BPH-1 cells but found that these cells did not form tumours even when co-injected with PZyoung or PZ-old stromal cells. For the in vivo coculture assay, we therefore used BPH-1 cells selected by long-term passage in culture. BPH-1 cells of high passage $(>100)$ injected alone still did not form 'tumours' before 3 weeks; these cells only formed small nodules in three of five animals after 8 weeks. Injection of high-passage BPH-1 cells with stromal cells from PZ-young donors accelerated 'tumour' formation, reducing the latency of tumour formation to 2 weeks, and three of the five mice developed 'tumours' (Figure $4 \mathbf{a}$ and $\mathbf{b}$ ). Extended observation showed that tumours in the control and BPH-1/PZ-young coinjection groups did not grow bigger, even after 12 weeks (data not shown). In contrast, when BPH-1 cells were injected together with an equal number of PZ-old stromal cells, all five nude mice developed larger 'tumours' with a shorter latency of 2 weeks. The average size of 'tumours' in mice injected with BPH-1/PZold cells was 1.8 -fold of those in the BPH-1/PZ-young group. The $\mathrm{H} \& \mathrm{E}$ staining shown in Figure $4 \mathrm{c}$ demonstrates that the BPH-1/PZold 'tumours' had more malignant cellular differentiation than did the tumours in the mice of the BPH-1/PZ-young group. By Ki67 IHC staining, the BPH-1/PZ-old 'tumours' also showed more proliferating cells than other groups (Figure $4 \mathbf{d}$ and $\mathbf{e}$ ).

Age-related stromal cells enhance tumour progression in vivo We also assessed the effect of the presence of stromal cells on the progression of tumours caused by PC-3 cells in nude mice. All sites inoculated with PC-3 cells developed tumours; mice in the PC-3/PZold group developed much larger tumours after 6 weeks than did the animals in the other two groups (Figure $5 \mathbf{a}$ and $\mathbf{b}$ ). Figure $\mathbf{5 c}$ shows that the PC-3 cells forming the tumours showed a similar high degree of atypia whether injected alone or in combination with stromal cells of different ages. As expected, the PC-3/PZ-old tumours showed more Ki67-positive proliferating cells in the IHC staining assays, suggesting increased aggressiveness (Figure 5d and e). These results show that stromal cells from aged donors strongly facilitated the hyperproliferation and neoplastic progression of PC-3 cells in vivo.

\section{Gene expression profiling analysis}

To probe the possible mechanism of ageing-related changes in prostate PZ stromal cells, we performed cDNA microarray analysis to measure differential gene expression. Using a fold change cutoff of $\geqslant 2$ or $\leqslant 0.5$, we identified 509 upregulated genes and 188 downregulated genes in PZ-old cells. Hierarchical cluster analysis revealed that PZ-young and PZ-old stromal cells showed distinctly different gene expression patterns (Figure 6a). The age-dependent differentially expressed genes could be grouped into several functional clusters including, among others, cell cycle/adhesion/apoptosis, immune response/inflammation, oxidative stress, and protein synthesis and degradation. Using Gene Ontology analysis, we identified a subset of 59 of the upregulated genes in PZ-old cells as genes coding for extracellular functional factors (Figure 6b). We selected several genes encoding extracellular paracrine mediators based on their known biological functions ${ }^{15-20}$ and verified their identities using Q-PCR. As 


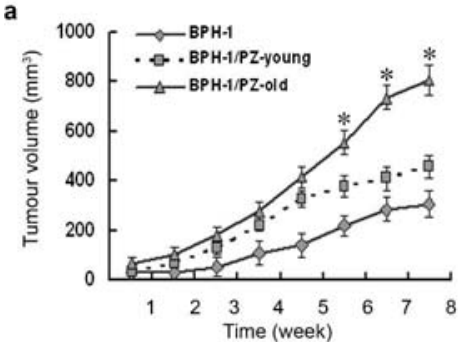

e

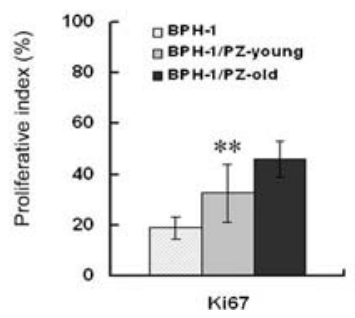

BPH-1
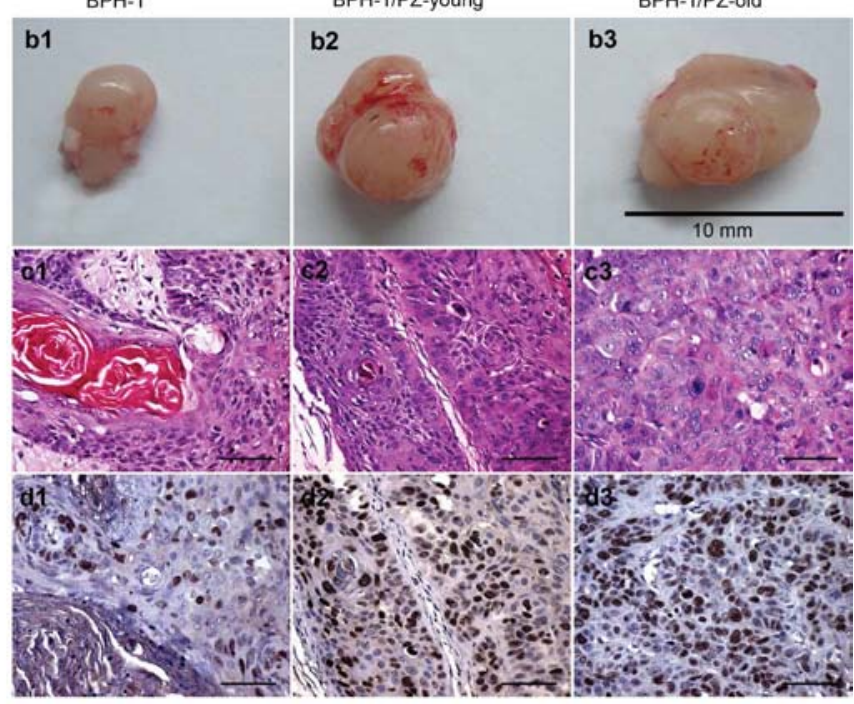

BPH-1/PZ-young

b2

BPH-1/PZ-old

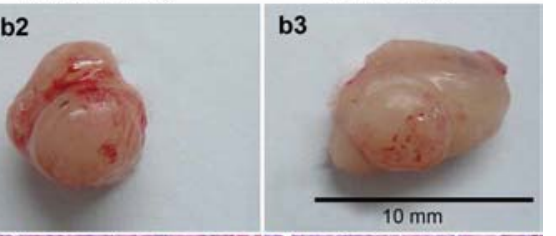

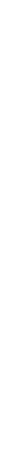

Figure 4 Effect of prostate stromal cells on tumourigenicity of BPH-1 in athymic mice. (a) Growth curves of the xenografts. 'Tumour' sizes were recorded weekly after inoculation until the experiments were terminated at 8 weeks. Tumour volume $=$ width $\times$ length ${ }^{2} \times 0.52$. Data are presented as mean \pm s.d.. $* P<0.01$, compared with the other two groups. (b) Gross appearance of tissue xenografts prepared with BPH-1 cells alone or with mixtures of BPH-1/PZ-young and BPH-1/PZ-old cells, and harvested from male nude mice after 8 weeks of growth. (c) Histology of 'tumour' sections from cocultured mouse models (H\&E staining). Control BPH-1 cells inoculated alone showed the prominent appearance of many concentric 'keratin pearls' (c1); 'tumour' grafts from the BPH-1/PZ-young group showed small foci or cords of epithelium (c2), while cells in the BPH-1/PZ-old group displayed a much more undifferentiated phenotype with amphophilic cytoplasm and nuclear pleiomorphism with one or several prominent nucleoli (c3). Scale bars $=40 \mu \mathrm{m}$. (d) Expression of Ki67 as assessed by immunostaining of 'tumour' xenografts formed by BPH1 cells (d1), BPH-1/PZ-young (d2) and BPH-1/PZ-old (d3) cocultured groups. Brown nuclear staining indicates a positive result. Scale bars=40 $\mu$ m. (e) The expression ratio of Ki67 indicated that the proliferative status of cells in the BPH-1/PZ-old group was much higher than that of cells in the other two groups. $* * P<0.05$, compared with other two groups. BPH, H\&E, haematoxylin and eosin; PZ, peripheral zone.

a
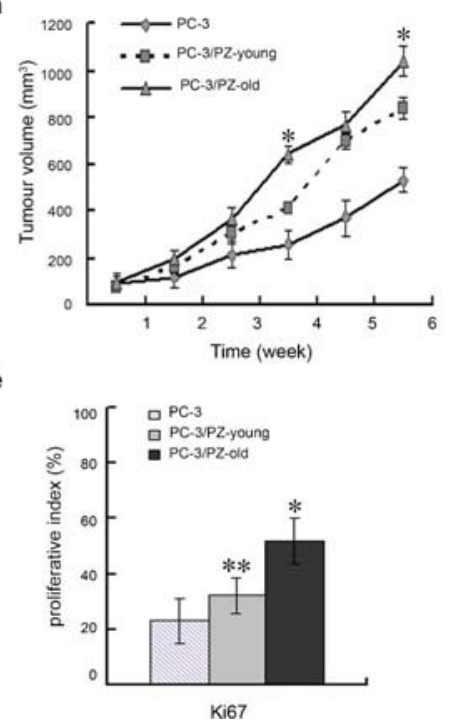

PC-3

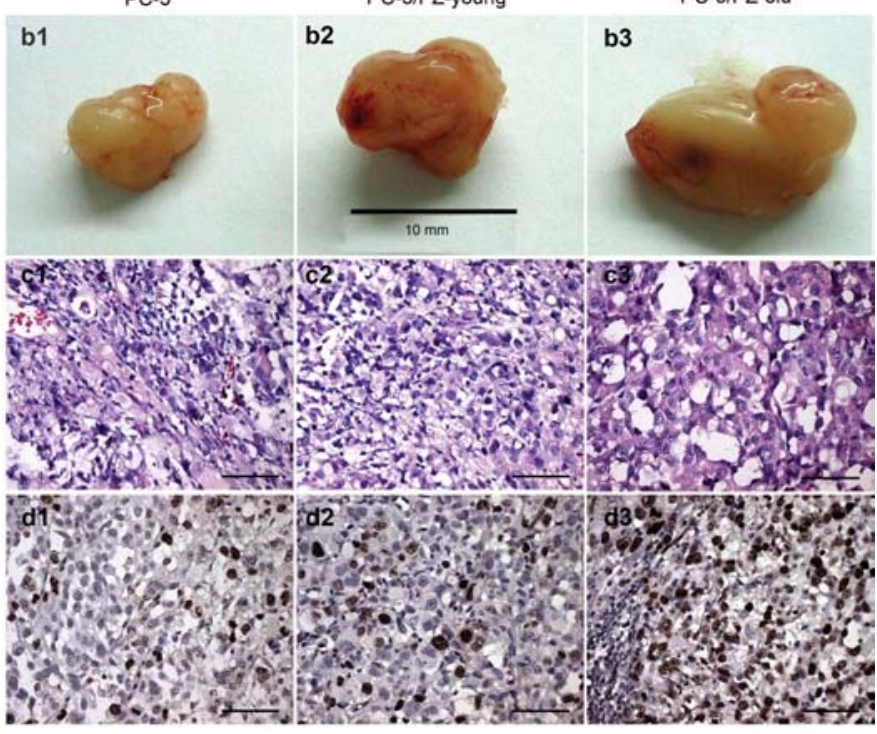

Figure 5 Effect of prostate stromal cells on tumour progression of PC-3 cells in athymic mice. (a) Growth curves of the xenografts. Tumour sizes were recorded weekly after inoculation until the experiments were terminated at 6 weeks. Tumour volume $=$ width $\times$ length ${ }^{2} \times 0.52$. Data are presented as mean \pm s.d. $* P<0.01$, compared with the other two groups. (b) Gross appearance of tissue xenografts prepared with PC-3 cells alone or with mixtures of PC-3/PZ-young or PC-3/PZ-old cells and harvested from male nude mice after 6 weeks of growth. (c) Histology of tumour sections from cocultured mice models assessed by H\&E staining. All three groups (PC3 cultured alone, PC-3/PZ-young and PC-3/PZ-old cocultured groups) displayed similar pleomorphic tumour cells. Scale bars =40 $\mu$ m. (d) Tumour xenografts of PC-3 cells, PC-3/PZ-young and PC-3/PZ-old cocultured groups were stained with Ki67 antibody. Brown nuclear staining indicates a positive result. Scale bars $=40$ m. (e) The expression ratio of Ki67 indicated that the proliferative status of cells in the PC-3/PZ-old group was much higher than that of cells in the other groups. $* P<0.01$, compared with other two groups. **P<0.05, compared with $\mathrm{PC}-3$ group. H\&E, haematoxylin and eosin; PZ, peripheral zone. 
a

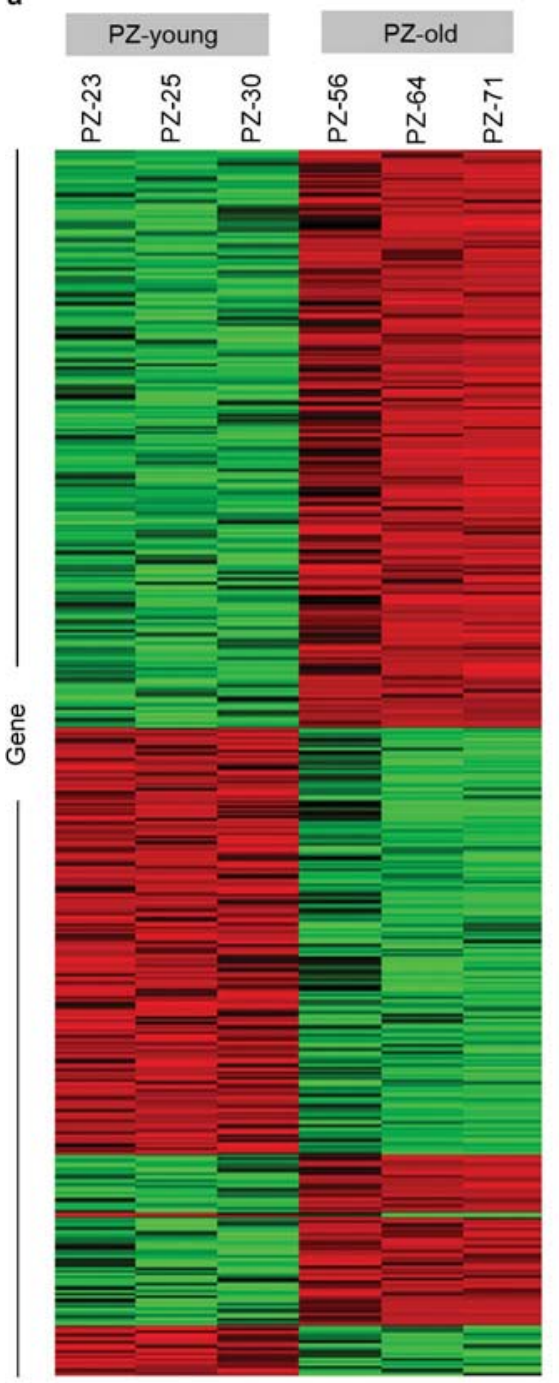

b

\section{PZ-young PZ-old}
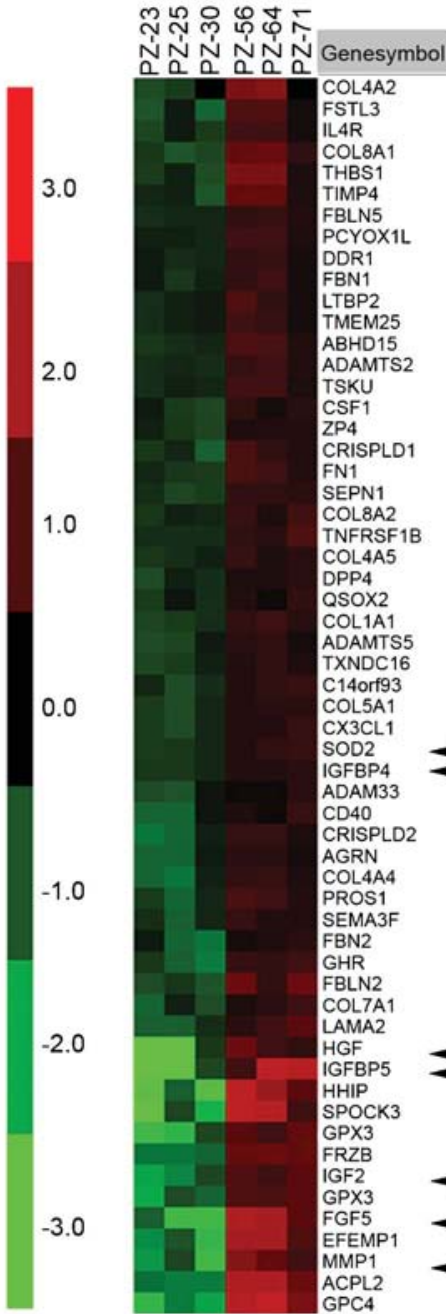

$-2.0$

\section{Gene description}

collagen, type IV, alpha 2

follistatin-like 3 (secreted glycoprotein)

interleukin 4 receptor

collagen, type VIII, alpha 1

Thrombospondin 1

opeptidase inhibitor 4

fibulin 5

prenylcysteine oxidase 1 like

discoidin domain receptor tyrosine kinase 1

inbrilin

latent transforming growth factor beta binding protein 2

transmembrane protein 25

abhydrolase domain containing 15

ADAM metallopeptidase with thrombospondin type 1

tsukushin

colony stimulating factor 1 (macrophage)

collucida glycoprotein 4

cysteine-rich secretory protein LCCL domain 1

fibronectin 1

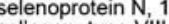

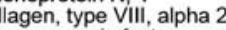

tumor necrosis factor receptor superfamily, member $1 \mathrm{~B}$ collagen, type IV, alpha

quiescin Q6 sulfhydryl o

a

collagen, type I, alpha 1

ADAM metallopeptidase with thrombospondin motif, 5

thioredoxin domain containing 16

chromosome 14 open reading frame 93

collagen, type $V$, alpha 1

chemokine (C-X 3 -C motif) ligand 1

chemokine (C-X3-C motif) ligand 1

-insulin-like growth factor binding protein 4

ADAM metallopeptidase domain 33

CD40 molecule. TNF receptor superfamily member 5

cysteine-rich secretory protein LCCL domain 2

collagen type IV alpha 4

protein S (alpha)

sema domain, immunoglobulin domain $(\mathrm{lg})$, secreted, $3 \mathrm{~F}$

fibrillin 2

growth hormone receptor

growth 2

VII, aipha 1

laminin, alpha 2

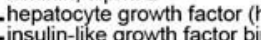

hedgehog interacting protein

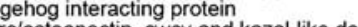

sparclosteonectin, cwcr and kaza

glutathione peroxidase 3 (plasma)

rizzled-related protein

(somatomedin A)

glutathione peroxidase 3 (plasma)

fibroblast growth factor 5

EGF-containing fibulin-like extracellular matrix protein 1

matrix metallopeptidase 1 (interstitial collagenase)

acid phosphatase-like 2

acid phosp

Figure 6 Hierarchical clustering analysis of differentially expressed genes in prostatic stromal cells from PZ-young and PZ-old donors. (a) Heatmap of all the differentially expressed genes classified based on fold change cutoff of $\geqslant 2$ or $\leqslant 0.5$. The relevant genes are grouped by hierarchical clustering analysis. Samples from two groups of PZyoung and PZ-old, each group including three separate individuals, are displayed in columns, and genes are displayed in rows. Most of the up- or down-regulated genes in PZ-old cells show opposite patterns in PZ-young cells, and there is good uniformity among the three different individuals in each group. (b) Heatmap of 59 genes encoding extracellular paracrine mediators that showed significant expression changes; these genes exhibit average fold changes of $\geqslant 2$ in the PZ-old group versus the PZ-young group. Arrows represent transcript alterations verified by Q-PCR. Gene expression is represented as a colour; the intensity of the colour correlates with fold change, with brighter red for higher values and brighter green for lower values, as shown in the colour bar in the middle. PZ, peripheral zone; Q-PCR, quantitative PCR.

shown in Table 1, there is good concordance between the results obtained from microarray and Q-PCR analysis.

\section{Measurement of paracrine factors and cytokines secreted by stromal cells}

Based on the results of microarray analysis, we used Western blot and ELISA to further analyse the secretion of four interesting soluble paracrine-acting factors by stromal cells. We observed significantly higher levels of secreted HGF, IGF2, IGFBP5 and MMP1 in PZ-old than in PZyoung cultures by Western blot (Figure 7a). The relative expression of these factors is shown in Figure $7 \mathbf{b}$. The ELISA results (Figure 7c) show that three of the four paracrine factors (HGF, IGFBP5 and MMP1) were secreted in higher amounts by PZ-old stromal cells than by PZ-young cells, the only exception being IGF2. The gene expression profiles were consistent at the protein and mRNA levels. These results suggest that the age-related phenotypic changes observed in prostate PZ stromal cells and their influences on epithelium were mediated, at least in part, by the increase of these paracrine factors.

\section{DISCUSSION}

Decades of research indicate that a permissive tissue microenvironment is required for somatic cells to reach full malignancy. ${ }^{21-25}$ Krtolica et al. ${ }^{25}$ reported that senescent fibroblasts were much more effective than presenescent cells at promoting proliferation and tumourigenesis of premalignant and malignant breast epithelial cells, providing a possible mechanistic link between stromal ageing and carcinogenesis. It has been reported that stromal cells play an important role in PCa initiation. ${ }^{9,26}$ Notably, the development of 
Table 1 Expression of selected differential genes (PZ-old versus PZyoung) coding for extracellular paracrine mediators. Changes are displayed as fold change from the microarray and were verified by Q-PCR

\begin{tabular}{llcc}
\hline & & \multicolumn{2}{c}{ Fold change } \\
\cline { 3 - 4 } Gene symbol & Gene description & Microarray & Q-PCR \\
\hline FGF5 & Fibroblast growth factor 5 & 13.4 & 2.2 \\
HGF & Hepatocyte growth factor & 12.8 & 9.6 \\
IGF2 & Insulin-like growth factor 2 & 6.6 & 9.4 \\
IGFBP4 & Insulin-like growth factor- & 2.1 & 7.1 \\
& $\quad$ binding protein 4 & & \\
IGFBP5 & Insulin-like growth factor- & 13.6 & 21.3 \\
& $\quad$ binding protein 5 & & 6.8 \\
MMP1 & Matrix metallopeptidase 1 & 4.5 & 5.5 \\
SOD2 & Superoxide dismutase 2 & 6.5 & \\
\hline
\end{tabular}

Abbreviations: PZ, peripheral zone; Q-PCR, quantitative PCR.

PCa preferentially occurs in the PZ of aged males, ${ }^{27}$ and cancers from the $\mathrm{PZ}$ are more aggressive. ${ }^{28}$ Our previous study characterized the growth of human prostate tissue, ${ }^{29}$ and it has been shown that $\mathrm{PZ}$ stromal cells have more promoting effects on epithelial tumour formation than transitional zone stromal cells. ${ }^{10}$ However, age-related alterations in PZ stromal cells and their contribution to PCa development are still poorly understood. Sensibar and collaborators ${ }^{14}$ demonstrated age-related changes of phenotype in prostate stromal cell cultures; a disadvantage of that study was that the aged stromal cells used were derived from benign prostatic hyperplasia $(\mathrm{BPH})$ and were thus not appropriate for the study of PCa.

In this study, we used a primary culture strategy to probe age-related changes in PZ stromal cells and to assess the possible effects of these changes on PCa initiation and progression. We isolated stromal cells from prostate $\mathrm{PZ}$ of donors of varying ages and found that the aged PZ stromal cells displayed enlarged and flattened morphological characteristics that represented features of senescence-associated differentiation. ${ }^{30,31}$ The existence of age-related cellular senescence was further verified by the observation of increased SA- $\beta$-GAL activity in the PZold fibroblasts. Immunocytochemical staining demonstrated that the cell types present in stromal cell cultures from PZ-young and PZ-old differed; PZ-young cell cultures were predominantly fibroblasts, but PZ-old cells were mixtures of fibroblasts and myofibroblasts. Our ultrastructural analysis also supported the conclusion that stromal cultures from PZ-old donors included cells that had undergone myofibroblast differentiation. The myogenically transformed fibroblasts were phenotypically and functionally similar to previously described 'cancer associated fibroblasts' ${ }^{32}$ Based on the fact that such myofibroblasts are more active than normal fibroblasts and can secrete aberrant factors that disrupt the architecture of the surrounding tissue and stimulate the proliferation of neighbouring epithelial cells, ${ }^{33,34}$ these changes might contribute to PCa development. Our results using PZ stromal cells from young and old donors indicate that there may be an inherent difference in prostate $\mathrm{PZ}$ stromal composition with increasing age.

The age-related changes in PZ stromal cells observed in this study had distinct influences on PCa development and progression. Using a three-dimensional coculture system, we found that aged PZ stromal cells were more effective than young PZ stromal cells in promoting the proliferation, migration and invasion of prostate epithelial cells. One interesting finding arising from our in vivo tissue recombination assays was that age-related PZ stromal cells promoted 'tumour' development when cotransplanted with high-passage BPH-1 cells. Most of the recombinant grafts formed by BPH-1 cells alone or by

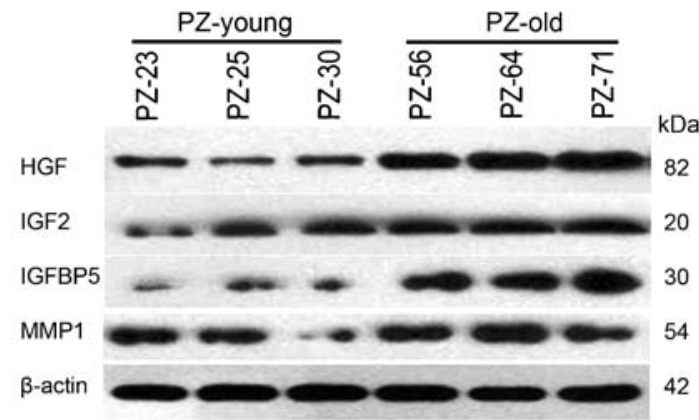

b
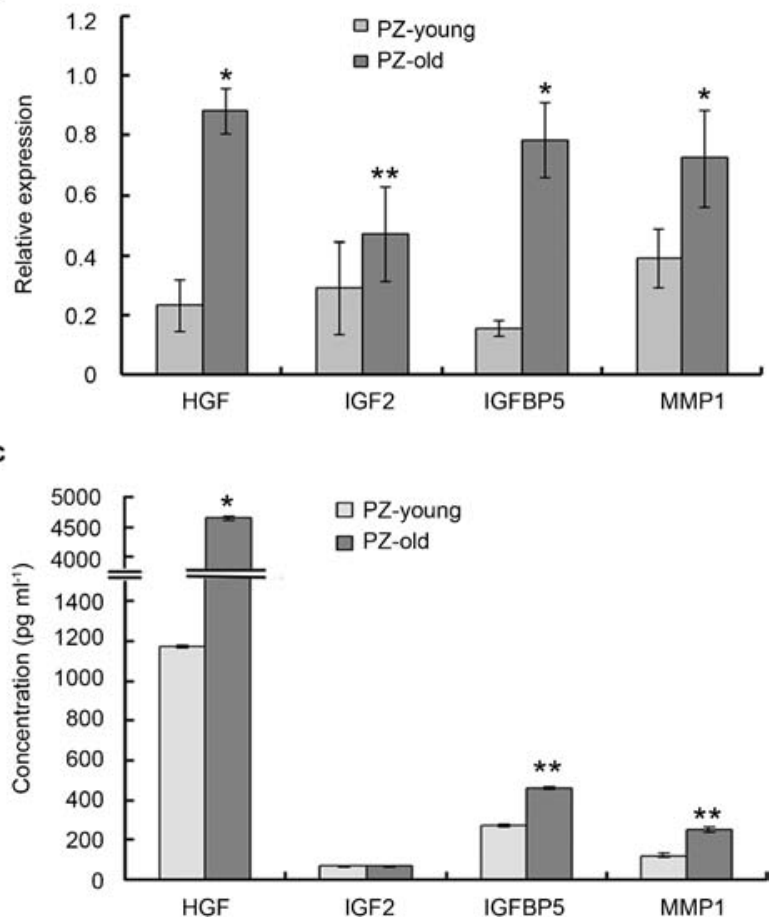

Figure 7 Determination of the levels of paracrine factors and cytokines in PZ-young and PZ-old stromal cells by Western blot and ELISA. (a) Representative Western blot bands were developed using antibodies against human HGF, IGF2, IGFBP5 and MMP1. $\beta$-actin served as a loading control. (b) Analysis of the relative expression of protein content by image intensity. Figures depict the intensity of the protein in representative samples from duplicate experiments displayed as composites of multiple Western blots. All band intensities were normalized to $\beta$-actin. (c) Concentrations of secreted HGF IGF2, IGFBP5 and MMP1 in stromal cell-conditioned medium determined by ELISA. The columns represent the mean values of three independent determinations \pm s.d. $* P<0.01, * * P<0.05$, compared with PZ-young. HGF, hepatocyte growth factor; IGF2, insulin-like growth factor 2; IGFBP5, insulin-like growth factor-binding protein 5; MMP1, matrix metallopeptidase 1; PZ, peripheral zone.

$\mathrm{BPH}-1 / \mathrm{PZ}$-young cells began to regress, with the appearance of many concentric 'keratin pearls' indicative of high-grade differentiation, at least in part suggesting their lower malignancy. However, in BPH-1/ PZ-old cocultured cells, the 'keratin pearls' almost disappeared and the 'tumour' cells became much more atypical. We also co-injected age-related PZ stromal cells and malignant PC-3 cells, and found that tumours of epithelial cells combined with PZ-old cells grew faster and attained larger size than tumours in control animals. This finding suggests that extracellular matrix factors produced by PZ-old cells might have enhanced growth stimulatory effects. 
In seeking a possible molecular explanation for the profound agerelated promoting effects of prostate stromal cells on epithelial cells, a number of factors must be considered. Bavik et al. induced senescent alterations in prostate fibroblasts by three different methods (oxidative stress, DNA damage and replicative exhaustion) and further identified a consistent program of gene expression that included a group of paracrine factors (e.g., FGF7, AREG and HGF) capable of influencing prostate epithelial growth in senescent cells. ${ }^{18}$ Their results suggested that the significant proliferative influence of the senescent fibroblasts on the epithelial cells was at least partially mediated through soluble factors. Recently, Coppe et al. ${ }^{35,36}$ further provided a large-scale characterisation of the senescence-associated secretory phenotype and identified many secretory factors including HGF, IGFBPs, MMPs, interleukins and others that are overexpressed in senescent fibroblasts. The altered secretory properties of senescent fibroblasts might be responsible for the observed promotion of malignant processes. ${ }^{19}$ However, the methods used in these studies to induce the senescence of stromal cells were artificial and might not reflect physiological 'senescence' in vivo.

A major advantage of our study is that it directly compares primary cultured prostate stromal cells from young and old donors. In the PZold specimens, we identified a series of upregulated genes associated with cancerous growth of the prostate, thus providing a probable connection between ageing and carcinogenesis. Among these genes, HGF is a powerful paracrine growth factor that affects a wide range of cellular processes, including cell differentiation, migration, angiogenesis and invasion in vitro and in vivo. ${ }^{15}$ The study of Nakashiro ${ }^{37}$ indicated that exogenous HGF injected at peritumour sites significantly enhanced PC-3 tumour growth in vivo. Naughton et al. ${ }^{38}$ observed that the serum HGF level in males with metastatic PCa is greatly elevated compared to those with or without localized cancer. In the present study, the paracrine factor that showed the greatest difference in expression between PZ-young and PZ-old groups was HGF. This observation suggests that the study of age-related differences in the levels of expression of paracrine secretory factors may be a fruitful subject for further investigation. Differences in the expression levels of other age-associated mitogenic factors, including IGF2, IGFBPs and MMP1, were also found in this study and have been reported by other researchers. ${ }^{20,35}$ IGF2 is a potent stimulator of mitogenesis and survival in many different cell types, ${ }^{39}$ and suppression of IGF2 expression in PCa cells leads to cell growth inhibition. ${ }^{40}$ The interaction between IGFs and IGFR triggers a signalling cascade (PI3K/Akt) that leads to cellular proliferation and inhibition of apoptosis. ${ }^{7}$ In vitro studies have further indicated that IGFBP5 could augment the activity of IGFs by facilitating ligand-receptor interaction and accelerating the progression of $\mathrm{PCa}$ in an androgen-depleted environment. ${ }^{41}$ Interestingly, an increasing number of IGF-independent actions of IGFBP5 in stimulating proliferation of various cells have recently been identified. ${ }^{42}$ The elevated levels of IGF2 and IGFBP5 observed in this study indicate that they may act in conjunction with other mitogenic factors (e.g., HGF) in prostate pathogenesis. Stromal cells are suggested to significantly contribute to the higher frequency of tumour invasion through an upregulated secretion of matrix-degrading metalloproteinases; this is known to induce cell proliferation and/or extracellular matrix degradation and occurs especially when stromal fibroblasts undergo senescence. ${ }^{43}$ The elevated level of MMP1 observed in PZ-old cells in the present study might explain the higher invasion index for PC-3 cells in our in vitro coculture system.

An inverse correlation between ageing and the growth rate of prostate stromal cells was found in our results and has also been reported in other studies. ${ }^{14}$ Age-associated cellular senescence might be the cause of decreased growth rates and morphological changes in cultured stromal cells in vitro. ${ }^{44,45}$ However, even though they grew more slowly, the PZ-old stromal cells in our study more effectively promoted PCa development and progression than the PZ-young cells. Phenotypic changes associated with ageing have been considered to be a result of the declining force of natural selection with age. In this view, cellular traits have been selected that maintain fitness in early life, but may promote cancer in aged organisms, a phenomenon termed evolutionary antagonistic pleiotropy. ${ }^{25,46}$

In summary, our results indicate that PZ-old stromal cells are more active than PZ-young stromal cells in promoting the malignant development of prostate epithelial cells and suggest that this might be due to the increased expression of extracellular paracrine mediators by PZ-old tissue. Our findings provide new insight into the biology of malignant processes in prostate tissue and suggest potential targets for the prevention or inhibition of PCa development and progression.

\section{AUTHOR CONTRIBUTIONS}

As principal investigators, YCW and SJX conceived the study, carried out the immunoassays, coculture assays and molecular genetic studies, and drafted the manuscript. SQY participated in carrying out the PCR assays, helped perform the statistical analysis and drafted the manuscript. XHW and YH participated in the collection of specimens and in primary cell culture. BMH and FJZ participated in the design and coordination of the study, and GHZ participated in the Western blot assays. All authors read and approved the final manuscript.

\section{COMPETING FINANCIAL INTERESTS}

The authors declare no competing financial interests.

\section{ACKNOWLEDGMENTS}

This work was supported by the Innovation Program of the Shanghai Municipal Education Commission (No. 102216) and by the National Natural Science Foundation of China (No. 81072096).

1 Jemal A, Siegel R, Xu J, Ward E. Cancer Statistics, 2010. CA Cancer J Clin 2010; 60: 277-300.

2 Ross RK, Pike MC, Coetzee GA, Reichardt JK, Yu MC et al. Androgen metabolism and prostate cancer: establishing a model of genetic susceptibility. Cancer Res 1998; 58: 4497-504.

3 Xia SJ, Hao GY, Tang XD. Androgen receptor isoforms in human and rat prostate. Asian J Androl 2000; 2: 307-10.

4 Xia SJ, Tang XD, Ma QZ. Androgen receptor isoforms in human prostatic cancer tissue and LNCaP cell line. Asian J Androl 2001; 3: 223-5.

5 Xia SJ, Xu CX, Tang XD, Wang WZ, Du DL. Apoptosis and hormonal milieu in ductal system of normal prostate and benign prostatic hyperplasia. Asian J Androl 2001; 3: $131-4$.

6 Sampson N, Untergasser G, Plas E, Berger P. The ageing male reproductive tract. J Pathol 2007; 211: 206-18.

7 Bosland MC. The role of steroid hormones in prostate carcinogenesis. J Nat/ Cancer Inst Monogr 2000; (27): 39-66.

8 Barclay WW, Woodruff RD, Hall MC, Cramer SD. A system for studying epithelialstromal interactions reveals distinct inductive abilities of stromal cells from benign prostatic hyperplasia and prostate cancer. Endocrinology 2005; 146: 13-8.

9 Cunha GR, Hayward SW, Wang YZ, Ricke WA. Role of the stromal microenvironment in carcinogenesis of the prostate. Int J Cancer 2003; 107: 1-10.

10 Zhao FJ, Han BM, Yu SQ, Xia SJ. Tumor formation of prostate cancer cells influenced by stromal cells from the transitional or peripheral zones of the normal prostate. Asian J Androl 2009; 11: 176-82.

11 Verona EV, Elkahloun AG, Yang J, Bandyopadhyay A, Yeh IT et al. Transforming growth factor-beta signaling in prostate stromal cells supports prostate carcinoma growth by upregulating stromal genes related to tissue remodeling. Cancer Res 2007; 67: 5737-46.

12 Livak KJ, Schmittgen TD. Analysis of relative gene expression data using real-time quantitative PCR and the method. Methods 2001; 25: 402-8. 
13 Zhang J, Hess MW, Thurnher M, Hobisch A, Radmayr C et al. Human prostatic smooth muscle cells in culture: estradiol enhances expression of smooth muscle cell-specific markers. Prostate 1997; 30: 117-29.

14 Sensibar JA, Pruden SJ, Kasjanski RZ, Rademaker A, Lee C et al. Differential growth rates in stromal cultures of human prostate derived from patients of varying ages. Prostate 1999; 38: 110-7.

15 Gmyrek GA, Walburg M, Webb CP, Yu HM, You X et al. Normal and malignant prostate epithelial cells differ in their response to hepatocyte growth factor/scatter factor. Am J Pathol 2001; 159: 579-90.

16 Guo N, Ye JJ, Liang SJ, Mineo R, Li SL et al. The role of insulin-like growth factor-II in cancer growth and progression evidenced by the use of ribozymes and prostate cancer progression models. Growth Horm IGF Res 2003; 13: 44-53.

17 Kaminski A, Hahne JC, Haddouti el-M, Florin A, Wellmann A et al. Tumour-stroma interactions between metastatic prostate cancer cells and fibroblasts. Int J Mol Med 2006; 18: 941-50.

18 Bavik C, Coleman I, Dean JP, Knudsen B, Plymate S et al. The gene expression program of prostate fibroblast senescence modulates neoplastic epithelial cel proliferation through paracrine mechanisms. Cancer Res 2006; 66: 794-802.

19 Coppe JP, Desprez PY, Krtolica A, Campisi J. The senescence-associated secretory phenotype: the dark side of tumor suppression. Annu Rev Pathol 2010; 5: 99-118.

20 Kuilman T, Peeper DS. Senescence-messaging secretome: SMS-ing cellular stress. Nat Rev Cancer 2009; 9: 81-94.

21 DePinho RA. The age of cancer. Nature 2000; 408: 248-54.

22 Liotta LA, Kohn EC. The microenvironment of the tumour-host interface. Nature 2001; 411: 375-9.

23 Coussens LM, Werb Z. Inflammation and cancer. Nature 2002:420: 860-7.

24 Krtolica A, Campisi J. Cancer and aging: a model for the cancer promoting effects of the aging stroma. Int J Biochem Cell Biol 2002; 34: 1401-14.

25 Krtolica A, Parrinello S, Lockett S, Desprez PY, Campisi J. Senescent fibroblasts promote epithelial cell growth and tumorigenesis: a link between cancer and aging. Proc Natl Acad Sci USA 2001; 98: 12072-7.

26 Bhowmick NA, Neilson EG, Moses HL. Stromal fibroblasts in cancer initiation and progression. Nature 2004; 432: 332-7.

27 Chen ME, Johnston DA, Tang K, Babaian RJ, Troncoso P. Detailed mapping of prostate carcinoma foci: biopsy strategy implications. Cancer 2000; 89: 1800-9.

28 Pavelic J, Zeljko Z, Bosnar MH. Molecular genetic aspects of prostate transition zone lesions. Urology 2003; 62: 607-13.

29 Xia SJ, Xu XX, Teng JB, Xu CX, Tang XD. Characteristic pattern of human prostatic growth with age. Asian J Androl 2002; 4: 269-71.

30 Ben-Porath I, Weinberg RA. The signals and pathways activating cellular senescence. Int J Biochem Cell Biol 2005; 37: 961-76.

31 Itahana K, Campisi J, Dimri GP. Mechanisms of cellular senescence in human and mouse cells. Biogerontology 2004; 5: 1-10.
32 Olumi AF, Grossfeld GD, Hayward SW, Carroll PR, Tlsty TD et al. Carcinomaassociated fibroblasts direct tumor progression of initiated human prostatic epithelium. Cancer Res 1999; 59: 5002-11.

33 Tuxhorn JA, Ayala GE, Smith MJ, Smith VC, Dang TD et al. Reactive stroma in human prostate cancer: induction of myofibroblast phenotype and extracellular matrix remodeling. Clin Cancer Res 2002; 8: 2912-23.

34 Gerdes MJ, Larsen M, Dang TD, Ressler SJ, Tuxhorn JA et al. Regulation of rat prostate stromal cell myodifferentiation by androgen and TGF-beta1. Prostate2004; 58: 299_ 307.

35 Coppe JP, Patil CK, Rodier F, Sun Y, Munoz DP et al. Senescence-associated secretory phenotypes reveal cell-nonautonomous functions of oncogenic RAS and the p53 tumor suppressor. PLoS Biol 2008; 6: 2853-68.

36 Coppe JP, Patil CK, Rodier F, Krtolica A, Beausejour CM et al. A human-like senescence-associated secretory phenotype is conserved in mouse cells dependent on physiological oxygen. PLoS One 2010; 5: e9188.

37 Nakashiro K, Okamoto M, Hayashi Y, Oyasu R. Hepatocyte growth factor secreted by prostate-derived stromal cells stimulates growth of androgen-independent human prostatic carcinoma cells. Am J Pathol 2000; 157: 795-803.

38 Naughton M, Picus J, Zhu X, Catalona WJ, Vollmer RT et al. Scatter factor-hepatocyte growth factor elevation in the serum of patients with prostate cancer. J Urol 2001 , 165: 1325-8.

39 Werner S, Grose R. Regulation of wound healing by growth factors and cytokines. Physiol Rev 2003; 83: 835-70.

40 Xu ZD, Oey L, Mohan S, Kawachi MH, Lee NS et al. Hammerhead ribozyme-mediated cleavage of the human insulin-like growth factor-II ribonucleic acid in vitro and in prostate cancer cells. Endocrinology 1999; 140: 2134-44.

41 Miyake H, Pollak M, Gleave ME. Castration-induced up-regulation of insulin-like growth factor binding protein- 5 potentiates insulin-like growth factor-I activity and accelerates progression to androgen independence in prostate cancer models. Cance Res 2000: 60: 3058-64.

42 Mohan S, Baylink DJ. IGF-binding proteins are multifunctional and act via GFdependent and -independent mechanisms. J Endocrinol 2002; 175: 19-31.

43 Egeblad M, Werb Z. New functions for the matrix metalloproteinases in cancer progression. Nat Rev Cancer 2002; 2: 161-74.

44 Dimri GP, Lee X, Basile G, Acosta M, Scott G et al. A biomarker that identifies senescent human cells in culture and in aging skin in vivo. Proc Natl Acad Sci USA 1995; 92: 9363-7.

45 Pendergrass WR, Lane MA, Bodkin NL, Hansen BC, Ingram DK et al. Cellular proliferation potential during aging and caloric restriction in rhesus monkeys (Macaca mulatta). J Cell Physiol 1999; 180: 123-30.

46 Campisi J. Aging, tumor suppression and cancer: high wire-act! Mech Ageing Dev 2005; 126: $51-8$ 